\title{
Linear increase of structural and numerical chromosome 9 abnormalities in human sperm regarding age
}

\author{
Mercè Bosch ${ }^{1}$, Osvaldo Rajmil ${ }^{2}$, Josep Egozcue ${ }^{3}$ and Cristina Templado ${ }^{\star 1}$
}

\begin{abstract}
${ }^{1}$ Departament de Biologia Cel.lular, Fisiologia i Immunologia, Facultat de Medicina, Universitat Autònoma de Barcelona, Bellaterra 08193, Spain; ${ }^{2}$ Servei d'Andrologia, Fundació Puigvert, Barcelona 08025, Spain; ${ }^{3}$ Departament de Biologia Cel.lular, Fisiologia i Immunologia, Facultat de Ciències, Universitat Autònoma de Barcelona, Bellaterra 08193, Spain
\end{abstract}

A simultaneous four-colour fluorescence in situ hybridisation (FISH) assay was used in human sperm in order to search for a paternal age effect on: (1) the incidence of structural aberrations and aneuploidy of chromosome 9, and (2) the sex ratio in both normal spermatozoa and spermatozoa with a numerical or structural abnormality of chromosome 9 . The sperm samples were collected from $\mathbf{1 8}$ healthy donors, aged 24-74 years (mean 48.8 years old). Specific probes for the subtelomeric $9 q$ region (9qter), centromeric regions of chromosomes 6 and 9, and the satellite III region of the Y chromosome were used for FISH analysis. A total of 190117 sperms were evaluated with a minimum of 10000 sperm scored from each donor. A significant linear increase in the overall level of duplications and deletions for the centromeric and subtelomeric regions of chromosome $9(P \leq 0.002)$, chromosome 9 disomy $(P<0.0001)$ as well as diploidy $(P<0.0001)$ was detected in relation to age. The percentage of increase for each 10-year period was $29 \%$ for chromosome 9 disomy, $18.8 \%$ for diploidy, and ranged from 14.6 to $28 \%$ for structural aberrations. Our results indicate a linear increase in structural aberrations and disomy for chromosome 9 in sperm with respect to age.

European Journal of Human Genetics (2003) 11, 754-759. doi:10.1038/sj.ejhg.5201049

Keywords: paternal age; spermatozoa; structural aberrations; aneuploidy; diploidy; sex ratio; chromosome 9; FISH

\section{Introduction}

Structural and numerical chromosome abnormalities are an important cause of miscarriage, infertility and congenital anomalies in humans. Aneuploidy is detected in $\sim 35 \%$ of spontaneous abortions and in $0.3 \%$ of newborns (for a review, see Hassold and $\mathrm{Hunt}^{1}$ ), while structural chromosome aberrations are found in $\sim 2 \%$ of sponta-

\footnotetext{
*Correspondence: C Templado, Departament de Biologia, Fisiologia i Immunologia, Facultat de Medicina, Universitat Autònoma de Barcelona, 08193 Bellaterra, Barcelona, Spain. Tel: + $3493-5813569$ Fax: + 34935811025 .

E-mail: cristina.templado@uab.es

Received 5 February 2003; revised 29 April 2003; accepted 7 May 2003
}

neous abortions and in $\sim 0.6 \%$ of live births (reviewed by $\mathrm{Jacobs}^{2}$ ).

The parental origin of chromosomal abnormalities seems to depend on the type of anomaly: while autosomal aneuploidies are mainly maternal in origin, $84 \%$ of de novo structural aberrations are paternal in origin. ${ }^{3}$ This preponderance of structural chromosome aberrations of paternal origin could be the result of a high frequency of structural lesions during spermatogenesis due to the absence of DNA repair in spermatids and spermatozoa. ${ }^{4}$ Despite the high paternal contribution to de novo rearrangements compared to the low frequency of paternally derived numerical abnormalities, the information on structural chromosome aberrations in sperm is more limited than 
that on numerical chromosome abnormalities (reviewed by Templado et $\mathrm{al}^{5}$ and Shi and Martin ${ }^{6}$ ). Some authors ${ }^{7,8}$ have described a positive age effect on structural chromosome abnormalities in human sperm karyotypes obtained by the human sperm-hamster oocyte fusion method. However, the number of sperm complements analysed in these studies was low due to the low efficiency of the human-hamster system.

Most fluorescence in situ hybridisation (FISH) studies have been carried out to determine if the frequency of aneuploid sperm increases with donor age. Chromosomes $1,{ }^{9,10} 6,{ }^{11} 8,{ }^{12} 9,{ }^{13} 12,{ }^{9} 13,{ }^{10} 14,{ }^{14} 18,,^{13,15,16} 21,{ }^{10,11,14}$ and the sex chromosomes ${ }^{9,11-13,15-17}$ have been investigated for an age effect, with seven of nine studies analysing sex chromosome aneuploidies. No age effect was observed in the disomy frequencies for chromosomes $6,8,9,12,13,14$, and controversial results were reported for chromosomes 1 , 21 , the sex chromosomes, and diploidy.

Up to now, only one study has been performed in spermatozoa searching simultaneously for structural and numerical abnormalities of chromosome 1 by three-colour FISH in 18 men aged $23-58$ years. ${ }^{10}$ The authors reported a positive significant association between donor age and the frequency of centromeric deletions of chromosome 1.

Chromosome 9 displays a high susceptibility to be broken. Pericentric inversion inv(9)(p11q13) is one of the most common chromosome heteromorphisms with a frequency of $\sim 1 \%$ in the general population, ${ }^{18}$ and chromosome 9 is frequently involved in reciprocal translocations in humans. ${ }^{19}$ Furthermore, this chromosome is more represented in unrejoined breakage events than expected by chance in human sperm complements. ${ }^{20,21}$ To our knowledge, no FISH studies regarding the effect of age on structural aberrations of chromosome 9 in sperm have been performed, and only one recent sperm study ${ }^{13}$ has been carried out to determine the incidence of numerical aberrations for chromosome 9 in relation to age using this technique.

The purpose of this study is to determine if donor age is associated with (1) structural 9q aberrations and disomy for chromosome 9 and (2) changes in the sex ratio in both normal sperm and sperm carrying a structural chromosome 9 abnormality. Approximately 200000 spermatozoa from 18 healthy sperm donors aged 24-74 years (mean 48.8 years old) have been analysed by four-colour FISH using specific probes for chromosomes 6 (CEP 6), 9 (CEP 9 and Telvysion 9q) and Y (Y sat III).

\section{Material and methods Sperm donors}

Semen samples were obtained from 18 healthy sperm donors aged 24-74 years, with no history of exposure to any known mutagens, clastogens, radiation or drugs. Of them, 14 were of proven fertility (CM2, CM6, C7, C8, CM7,
CM8, CM9, CM10, CM11, CM12, CM13, CM14, CM15, CM16) and the remaining four were of unknown fertility. Only four of them were smokers (CM1, CM4, CM6, CM10). All donors gave informed consent and the study was approved by our institutional Ethics Committee. Numerical abnormalities for chromosomes $6,21, \mathrm{X}$ and $\mathrm{Y}$ in the sperm of all men have already been reported by us. ${ }^{11}$

\section{Sperm preparation and FISH}

Sperm fixation, sperm nuclei decondensation and hybridisation procedures were performed as described. ${ }^{11}$

\section{Probes}

Specific probes for the centromeric regions of chromosomes 6 (1:1 mix of Spectrum green, CEP 6, and Spectrum orange, CEP 6; Vysis, Inc.; Downers Grove, IL, USA), and 9 (Spectrum green, CEP 9, Vysis, Inc.), a DNA satellite III probe for the $\mathrm{Y}$ chromosome (Spectrum aqua, Y sat III; Vysis, Inc.) and a subtelomeric probe for the long arm of chromosome 9 (Spectrum orange, Telvysion 9q, Vysis, Inc.) were used for the four-colour FISH study. Chromosome 6 provided an internal control to distinguish between disomy for 9 and $\mathrm{Y}$ chromosomes and a diploid cell, because in diploid cells two copies for chromosome 6 would be seen.

\section{Data collection and scoring criteria}

Only slides with a hybridisation efficiency of at least $99 \%$ were scored and only intact and nonoverlapped sperm nuclei identified as decondensed sperm, because of the presence of a tail, were evaluated. Standard scoring criteria, previously described, ${ }^{11}$ were followed to evaluate numerical and structural chromosome abnormalities. The presence of two signals for $9 \mathrm{q}$ or 9 cen regions was scored as structural duplications for $9 \mathrm{q}$ or 9 cen regions, respectively. Structural deletions for $9 \mathrm{q}$ or 9 cen were considered when no signals for these regions were detected.

The absence of a Y fluorescence signal could be expected in normal X-bearing spermatozoa as well as in nullisomic spermatozoa for the $\mathrm{Y}$ chromosome. Owing to the low number of this type of cell, we assume an underestimation of nullisomy Y. All slides were scored by the same person and ambiguous signals were examined, at least, by a second, independent observer. In total, 50\% of all slides were coded previously to scoring, and donor age and identities were unavailable to the scorer until after data collection was completed.

\section{Statistical analysis}

The data sets were fitted using Poisson's regression with age as the only covariate, checking linear and quadratic trends. In the situations where the deviance goodness-of-fit test detected an inadequateness of the Poisson assumption, the negative binomial distribution was then employed to perform the statistical tests. The estimated percentage 
increase for a period of 10 years jointly with its 95\% confidence interval was also calculated for each abnormality from the coefficient of the linear model. In order to check the adequacy of the models and to get an overview of the results, the predicted and observed proportions were plotted. The statistical analyses were performed by using the GENMOD procedure of the SAS statistical package.

$\chi 2$ (with Yates correction) with one degree of freedom was used to determine whether the gametic sex ratio in sperm carrying a structural chromosome abnormality for chromosome 9 deviated significantly from 1 , and to compare frequencies between structural abnormalities of 9cen and 9q.

\section{Results}

A four-colour FISH assay was used to detect simultaneously structural aberrations for $9 \mathrm{q}$, disomy for chromosome 9, diploidy and the sex ratio in human sperm. A total of 190117 spermatozoa from 18 normal donors were analysed.

Table 1 summarises the frequencies of structural chromosome 9 aberrations observed in our donors, and Table 2 shows the individual sex ratio, disomy for chromosomes 6 , 9 and $\mathrm{Y}$, and diploidy frequencies.

Interindividual heterogeneity was found in the incidence of all types of structural abnormalities of chromosome 9 (duplications or deletions) as well as in the frequencies of 9 disomy and diploidy. The linear regression test for trends used to analyse the data only considers variability caused by age, underestimating the overdispersion not explained by age, and probably related to other factors. When a significant heterogeneity was found with this test, an additional model based on the negative binomial distribution was used to account for it.

Our results indicate a significant linear tendency to an increase in relation to age in the overall level of partial duplications of chromosome 9 (9cen, $P<0.0001$, and 9qter, $P=0.002$ ), partial deletions (9cen, $P=0.001$, and 9qter, $P<0.0001)$, disomy $9(P<0.0001)$, and diploidy $(P<0.0001)$. Duplications of $9 \mathrm{q}$ were significantly more frequent than 9cen duplications $(P=0.017)$. For each donor, the ratio of $\mathrm{X}$ - to Y-bearing sperm was $1: 1$, as expected. X-bearing sperm carrying a structural abnormality of chromosome 9 was significantly more frequent than Y-bearing sperm carrying the same chromosome aberration $(P=0.041)$. When the model based on a negative binomial distribution was assumed, an increase for all chromosome abnormalities studied was observed for each 10-year interval (Table 3).

The linear trends relating age, diploidy, disomy and structural abnormality frequencies for chromosome 9 are represented in Figure 1.

\section{Discussion}

In this work we were specifically interested in analysing simultaneously if numerical and structural chromosome 9 abnormalities in human sperm are influenced by donor age in a series of 18 individuals selected on the basis of age. Chromosome 9 was chosen because of its high incidence of breaks shown in somatic cells and spermatozoa. ${ }^{20,21}$ We also investigated whether chromosome 9 structural or numerical abnormalities influenced by donor age were more likely to occur in X- or Y-bearing sperm.

Table 1 Sperm structural chromosome 9 aberrations in 18 normal men

\begin{tabular}{|c|c|c|c|c|c|c|c|}
\hline \multirow{2}{*}{ Donor } & \multirow[b]{2}{*}{ Age (years) } & \multicolumn{3}{|c|}{ Structural duplications (\%) } & \multicolumn{3}{|c|}{ Structural deletions (\%) } \\
\hline & & $9 c$ & 9qtel & Total & $9 c$ & 9qtel & Total \\
\hline CM1 & 24 & 0.07 & 0.07 & 0.13 & 0.08 & 0.08 & 0.15 \\
\hline $\mathrm{CM} 3$ & 24 & 0.04 & 0.08 & 0.11 & 0.09 & 0.07 & 0.16 \\
\hline CM4 & 25 & 0.03 & 0.13 & 0.16 & 0.09 & 0.05 & 0.13 \\
\hline CM7 & 30 & 0.08 & 0.05 & 0.13 & 0.05 & 0.03 & 0.07 \\
\hline CM5 & 37 & 0.04 & 0.09 & 0.13 & 0.06 & 0.07 & 0.13 \\
\hline CM2 & 38 & 0.04 & 0.05 & 0.08 & 0.09 & 0.07 & 0.17 \\
\hline CM6 & 42 & 0.05 & 0.11 & 0.15 & 0.08 & 0.02 & 0.10 \\
\hline C7 & 45 & 0.00 & 0.07 & 0.07 & 0.03 & 0.03 & 0.06 \\
\hline C8 & 49 & 0.10 & 0.11 & 0.22 & 0.08 & 0.07 & 0.15 \\
\hline CM8 & 52 & 0.08 & 0.08 & 0.16 & 0.08 & 0.04 & 0.11 \\
\hline CM9 & 54 & 0.16 & 0.18 & 0.35 & 0.15 & 0.14 & 0.30 \\
\hline CM10 & 56 & 0.15 & 0.16 & 0.31 & 0.13 & 0.15 & 0.28 \\
\hline CM11 & 60 & 0.16 & 0.15 & 0.31 & 0.06 & 0.15 & 0.22 \\
\hline CM12 & 62 & 0.08 & 0.13 & 0.21 & 0.09 & 0.10 & 0.19 \\
\hline CM13 & 64 & 0.09 & 0.17 & 0.26 & 0.21 & 0.12 & 0.33 \\
\hline CM14 & 71 & 0.13 & 0.11 & 0.24 & 0.10 & 0.11 & 0.21 \\
\hline CM15 & 72 & 0.12 & 0.15 & 0.27 & 0.13 & 0.18 & 0.31 \\
\hline CM16 & 74 & 0.11 & 0.11 & 0.22 & 0.17 & 0.19 & 0.36 \\
\hline Total mean & 48.8 & 0.08 & 0.11 & 0.20 & 0.10 & 0.09 & 0.19 \\
\hline
\end{tabular}


Table 2 Individual frequencies of disomy for chromosomes 6, 9 and Y, diploidy and sex ratio in spermatozoa from 18 normal men

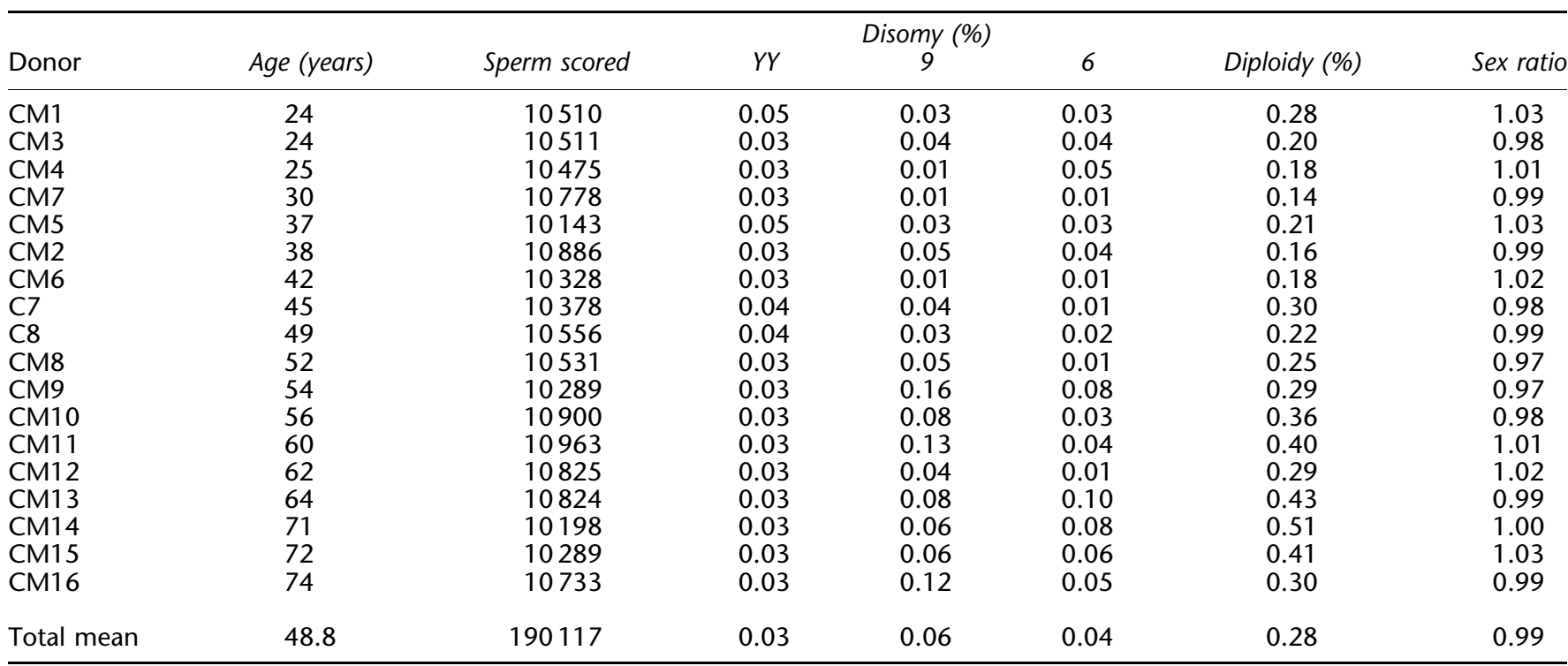

Table 3 Results of statistical analyses used to determine the association between male donor age and the rate disomy and structural chromosomal abnormalities for chromosome 9

\begin{tabular}{|c|c|c|c|c|c|}
\hline \multirow{3}{*}{ Chr. abnormality } & \multicolumn{4}{|c|}{ Linear trend in regression model } & \multirow{3}{*}{$\begin{array}{c}\text { Heterogeneity } \\
P \text {-value }\end{array}$} \\
\hline & $\%$ Increase for 10 years & & & P-value & \\
\hline & & Less & Upper & & \\
\hline \multicolumn{6}{|l|}{$\begin{array}{l}\text { Disomy } \\
9\end{array}$} \\
\hline & 29.0 & 15.0 & 44.6 & $<0.0001$ & $0.004^{b}$ \\
\hline \multicolumn{6}{|c|}{ Structural duplications } \\
\hline 9cen & 21.2 & 10.3 & 33.1 & $<0.0001$ & $0.003^{b}$ \\
\hline 9qter & 14.6 & 5.2 & 24.9 & 0.002 & 0.187 \\
\hline Total mean & 18.4 & 11.1 & 26.1 & $<0.0001$ & $0.003^{\mathrm{b}}$ \\
\hline \multicolumn{6}{|c|}{ Structural deletions } \\
\hline 9cen & 16.5 & 6.5 & 27.8 & 0.001 & 0.075 \\
\hline 9qter & 28.0 & 16.5 & 40.5 & $<0.0001$ & $0.037^{b}$ \\
\hline Total mean & 21.9 & 14.2 & 30.0 & $<0.0001$ & $0.001^{\mathrm{b}}$ \\
\hline
\end{tabular}

${ }^{a}$ Confidence interval.

${ }^{b}$ Negative binomial distribution was assumed.

We found increased frequencies of all types of structural chromosome 9 aberrations analysed (deletions and duplications of the 9cen and $9 \mathrm{q}$ regions) with regard to age. In a similar study of structural aberrations of chromosome 1 in men aged 23-58 years, McInnes et $a l^{10}$ found a donor age effect for the frequency of structural aberrations for centromeric deletions of this chromosome. In studies of human sperm chromosome complements, a donor age effect has also been found for structural chromosome aberrations. ${ }^{7,8}$ Martin and Rademaker ${ }^{7}$ observed a significant correlation between donor age and structural chromosome aberrations when they compared the data obtained from two donor age groups (donors aged 20-24 versus donors aged 45-55 years). Prestes Sartorelli et al ${ }^{8}$ found an increased frequency of structural aberrations in men of advanced age (59-74 years old), with respect to a control group (23-29 years old).

The association between age and structural chromosome abnormalities could be due to the high number of spermatid divisions per year, to a decreased effectiveness of DNA reparation systems and to an accumulation of DNA damage from environmental exposure. ${ }^{7}$

In agreement with the increased number of chromosome 9 lesions observed by us in relation to age, nonrandom 


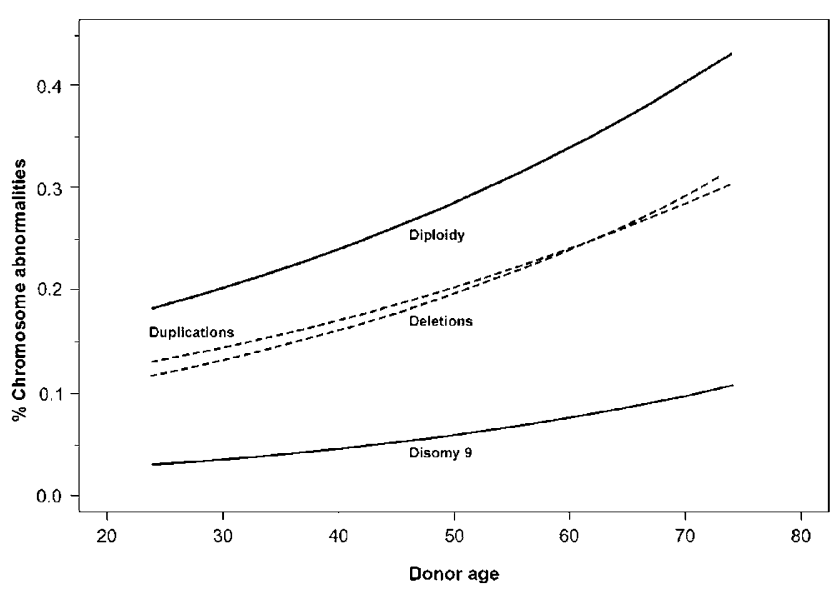

Figure 1 Relationship between age and frequencies of numerical and structural abnormalities for chromosome 9, and diploidy.

distribution of breaks in this chromosome was previously reported in sperm karyotypes of normal men. ${ }^{4,20}$ These studies are coincident in that chromosome 9 has a higher number of breakpoints than expected by chance and also in that $50 \%$ of the breakpoints are located between the centromere and the $9 \mathrm{qh}+$ segment. In somatic cells, Starke et $\mathrm{al}^{22}$ proposed that the homology between $9 \mathrm{p} 12$ and $9 q 13-q 21.1$ with the short arms of the acrocentric human chromosomes, made these chromosomes more prone to chromosome rearrangements and increased the risk of an interchromosomal effect among them.

A significant excess of sperm bearing partial duplications of the $9 \mathrm{q}$ region compared with those bearing partial duplications of 9cen $(P=0.017)$ was detected. In a similar study in control donors, ${ }^{23}$ it was also observed that sperm carrying partial duplications and deletions of $1 \mathrm{p}$ were five times more frequent than those carrying 1cen structural abnormalities. Partial duplications and deletions may originate by: (1) structural rearrangements, (2) unequal crossover in meiosis I producing partial duplications and, (3) breakage events during meiosis. Unfortunately, the specific type of structural aberrations found could not be determined because the multicolour FISH technique in spermheads cannot discriminate among these aberrations. Despite what has previously been stated above, the excess of the duplications of $9 \mathrm{q}$ observed here could reflect an excess of acentric fragments in sperm, as described in studies of human sperm karyotypes, both in controls ${ }^{20,21,24}$ and aged series. ${ }^{7,8}$ Among sperm carrying chromosome 9 structural aberrations, a significant increase was found of sperm without a sex chromosome (assumed as an Xbearing sperm) compared with sperm bearing a Y chromosome $(P=0.042)$. Unfortunately, this excess of $\mathrm{X}$-bearing sperm could result from an underestimation of $\mathrm{X}$ chromosome nullisomy due to the absence of the $\mathrm{X}$ chromosome probe in this study. Nevertheless, in an epidemiological study of 4300 individuals from Japan, ${ }^{25}$ the incidence of inv (9) carriers was about two times higher in the female group than in the male group $(P<0.05)$.

Interestingly, an increased frequency of disomy for chromosome 9 related to age was found. In a similar study, Luetjens et al $^{13}$ also observed a higher percentage of 9 disomy in donors $>60$ years than in donors $<30$ years old, but this difference did not reach statistical significance $(P=0.061)$. In another two studies on chromosome 9 aneuploidy in spermatozoa published to date, using PRINS $^{26}$ or chromosome painting, ${ }^{27}$ the frequencies of disomy for this chromosome were similar to those for other autosomes. However, Martin and Rademaker, ${ }^{28}$ analysing human sperm karyotypes from normal men, found that chromosomes 9 and 21 were the only autosomes to show an increased level of hyperhaploidy. The reason for increasing frequencies of disomy of chromosome 9 associated with age still remains unclear, but a tendency of chromosomes 1, 9 and 16 to show higher frequencies of disomy than other autosomes has been reported in human sperm. ${ }^{29}$ Heterochromatin characteristically has lower recombination rates and this has been proposed as playing a crucial role in chromosome pairing. Furthermore, synaptonemal complexes are structurally different in euchromatic and heterochromatic regions, suggesting that homologous partner recognition is due to a higher level of chromosome organisation rather than to sequence homology. ${ }^{30,31}$ Differences of length of the C-heterochromatin of the two 9 chromosomes increase the risk for uneven pairing during meiosis I and, consequently, for meiotic nondisjunction. ${ }^{32,33}$

The frequencies of disomy, diploidy and structural chromosome abnormalities, at least for the chromosomes studied, show a high trend to increase with each 10-year interval, ranging from 12.4 to $29.0 \%$. The highest rate of increase corresponds to disomy 9 . With respect to structural aberrations, the highest percentage of increase corresponds to $9 \mathrm{q}$ deletions and centromeric duplications. These percentages of increase could be related to a progressively deteriorating testicular environment - host factors $^{34,35}$ - or to accumulated DNA damage due to environmental mutagens - external factors - in older men. Both causes could generate disturbances in the meiotic process leading to an increase of the expected frequencies of numerical and structural chromosome abnormalities.

In conclusion, a linear relationship between donor age and increasing frequencies of structural chromosome aberrations and disomy for chromosome 9, and diploidy in human spermatozoa have been herein demonstrated. Thus far, disomy frequencies for 11 of the 24 chromosomes of the human sperm karyotype have been evaluated by FISH in relation to age, while structural aberrations have only been analysed for chromosomes $1^{10}$ and 9 (present study). It would be necessary to include other 
chromosomes in these studies to define the real effect of advanced paternal age on chromosomal abnormalities in sperm.

\section{Acknowledgements}

We thank Dr Pere Puig Casado for performing the statistical analysis and also Mr Chuck Simmons for the revision and correction of the English of this manuscript. This work received financial support from Ministerio de Ciencia y Tecnología (Project BFI2002-01193) and Generalitat de Catalunya (CIRIT, 2001 SGR-00201), Spain.

\section{References}

1 Hassold T, Hunt PA: To err (meiotically) is human: the genesis of human aneuploidy. Nat Rev 2001; 2: 280-291.

2 Jacobs P: The chromosome complement of human gametes. Oxford Rev Reprod Biol 1992; 14: 48-72.

3 Olson SD, Magenis RE: Preferential paternal origin of de novo structural chromosome rearrangements; in Daniel A (ed): The cytogenetics of mammalian autosomal rearrangements. New York: Alan R Liss, 1988, pp 583-599.

4 Estop AM, Márquez C, Munne $S$ et al: An analysis of human sperm chromosome breakpoints. Am J Hum Genet 1995; 56: 452-460.

5 Templado C, Márquez C, Munne S et al: An analysis of human sperm chromosome aneuploidy. Cytogenet Cell Genet 1996; 74: $194-200$

6 Shi Q, Martin RH: Aneuploidy in human sperm: a review of frequency and distribution of aneuploidy, effects of donor age and lifestyle factors. Cytogenet Cell Genet 2000; 90: 219-226.

7 Martin RH, Rademaker AW: The effect of age on the frequency of sperm chromosomal abnormalities in normal men. Am J Hum Genet 1987; 41: 484-492.

8 Prestes Sartorelli EM, Mazzucatto LF, Monterio de Pina-Neto J: Effect of paternal age in human sperm chromosomes. Fertil Steril 2001; 79: 1119-1123.

9 Martin RH, Spriggs E, Ko E, Rademaker AW: The relationship between paternal age, sex ratios, and aneuploidy frequencies in human sperm, as assessed by multicolor FISH. Am J Hum Genet 1995; 57: 1395-1399.

10 McInnes B, Rademaker AW, Martin RH: Donor age and the frequency of disomy for chromosomes 1, 13, 21 and structural abnormalities in human spermatozoa using multicolor fluorescence in-situ hybridization. Hum Reprod 1998; 13: 2489-2494.

11 Bosch M, Rajmil O, Martínez-Pasarell O, Egozcue J, Templado C: Linear increase of diploidy in human sperm with age: a fourcolour FISH study. Eur J Hum Genet 2001; 9: 533-538.

12 Robbins WA, Baulch JE, Moore II D, Weier HU, Blakey D, Wyrobek AJ: Three-probe fluorescence in situ hybridisation to assess chromosome X, Y, and aneuploidy in sperm of 14 men from two healthy groups: evidence for a paternal age effect on sperm aneuploidy. Reprod Fertil Dev 1995; 7: 799-809.

13 Luetjens CM, Rolf C, Gassner P, Werny JE, Nieschlag E: Sperm aneuploidy rates in younger and older men. Hum Reprod 2002; 7: $1826-1832$.

14 Rousseaux S, Hazzouri M, Pelletier R, Monteil M, Usson Y, Sele B: Disomy rates for chromosomes 14 and 21 studied by fluorescent in-situ hybridization in spermatozoa from three men over 60 years of age. Mol Hum Reprod 1998; 4: 695-699.

15 Griffin DK, Abruzzo MA, Millie EA et al: Nondisjunction in human sperm: evidence for an age effect of increasing paternal age. Hum Mol Genet 1995; 4: 2227-2232.

16 Asada H, Sueoka K, Hashiba T, Kuroshima M, Kobayashi N, Yoshimura Y: The effect of age and abnormal sperm count on the nondisjunction of spermatozoa. I Assist Reprod Genet 2000; 17: $51-59$.

17 Kinakin B, Rademaker A, Martin R: Paternal age effect of YY aneuploidy in human sperm, as assessed by fluorescence in situ hybridization. Cytogenet Cell Genet 1997; 78: 116-119.

18 Gardner RJM, Sutherland GR: Chromosome abnormalities and genetic counseling. Oxford monograph on medical genetics. New York: Oxford University Press, 1996, Vol 29: pp 139-152.

19 Cohen O, Cans C, Cuillel M et al: Cartographic study: breakpoints in 1574 families carrying human reciprocal translocations. Hum Genet 1996; 97: 659-667.

20 Brandriff BF, Gordon LA, Moore II DH, Carrano AV: An analysis of structural aberrations in human sperm chromosomes. Cytogenet Cell Genet 1988; 47: 29-36.

21 Estop AM, Cieply K, Vankirt V, Munne S, Garver K: Cytogenetics studies in human sperm. Hum Genet 1991; 87: 447-451.

22 Starke H, Seidel J, Henn W et al: Homologous sequences at human chromosome 9 bands p12 and q13-21.1 are involved in different patterns of pericentric rearrangements. Eur J Hum Genet 2002; 10: $790-800$.

23 Sloter ED, Lowe X, Moore II DH, Nath J, Wyrobek AJ: Multicolor FISH analysis of chromosomal breaks, duplications, deletions, and numerical abnormalities in the sperm of healthy men. Am J Hum Genet 2000; 67: 862-872.

24 Benet J, Genescà A, Navarro J, Egozcue J, Templado C: Cytogenetic studies in motile sperm from normal men. Hum Genet 1992; 89: 176-180.

25 Yamada K: Population studies of inv(9) chromosomes in 4,300 Japanese: incidence, sex difference and clinical significance. Jpn J Hum Genet 1992; 37: 293-301.

26 Pellestor F, Girardet A, Coignet L, Andreo B, Charlieu JP: Assessment of aneuploidy for chromosomes 8, 9, 13, 16, and 21 in human sperm by using primed in situ labeling technique. Am J Hum Genet 1996; 58: 797-802.

27 Rives N, Mazurier S, Bellet D, Joly G, Mace B: Assessment of autosome and gonosome disomy in human sperm nuclei by chromosome painting. Hum Genet 1998; 102 $616-623$.

28 Martin RH, Rademaker A: The frequencies of aneuploidy among individual chromosomes in 6,821 human sperm complements. Cytogenet Cell Genet 1990; 53: 103-107.

29 Guttenbach M, Engel W, Schmid M: Analysis of structural and numerical chromosome abnormalities in sperm of normal men and carriers of constitutional chromosome aberrations. A review. Hum Genet 1997; 100: 1-21.

30 Gagne R, Laberge C, Tanguay R: Cytological aspect and intranuclear localization of the heterochromatic segments of C9 chromosomes in man. Chromosoma 1973; 41: 159-166.

31 Renaud H, Gasser SM: Heterochromatin: a meiotic matchmaker? Trends Cell Biol 1997; 7: 201-205.

32 Amiel A, Sardos-Albertini F, Fejgin MD, Sharony R, Diukman R, Bartoov B: Interchromosomal effect leading to an increase in aneuploidy in sperm nuclei in a man heterozygous for pericentric inversion (inv 9) and C-heterochromatin. J Hum Genet 2001; 46: $245-250$.

33 Serra A, Brahe C, Millington-Ward A et al: Pericentric inversion of chromosome 9: prevalence in 300 Down syndrome families and molecular studies of nondisjunction. Am J Med Genet 1990; 7: $162-168$.

34 Mroz K, Hassold TJ, Hunt PA: Meiotic aneuploidy in the XXY mouse: evidence that a compromised testicular environment increases the incidence of meiotic errors. Hum Reprod 1999; 14: $1151-1156$.

35 Egozcue S, Blanco J, Vendrell JM et al: Human male infertility: chromosome anomalies, meiotic disorders, abnormal spermatozoa and recurrent abortion. Hum Reprod Update 2000; 6: 93-105. 